\title{
Edema agudo hemorrágico en un neonato
}

\author{
R. Checa Rodríguez ${ }^{a}$, I. Carabaño Aguado ${ }^{b}$, B. Álvarez Fernández ${ }^{a}$
}

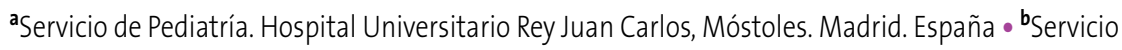
de Pediatría. Hospital General de Villalba, Hospital Universitario Rey Juan Carlos. Madrid. España.

Publicado en Internet: 11-marzo-2015

Raquel Checa Rodríguez: raquel_checa85@hotmail.com
Palabras clave:

- Edema agudo hemorrágico

- Vasculitis

- Púrpura de Schönlein-Henoch

- Exantema

- Neonato
El edema agudo hemorrágico del lactante (EAHL) es una enfermedad infrecuente de curso benigno, de aparición entre los tres meses y los dos años de edad. De forma excepcional debuta en el periodo neonatal. Se caracteriza por la aparición de lesiones cutáneas eritematosas y purpúricas de predominio en miembros inferiores y región facial, que tienden a la resolución espontánea en días o semanas.

Presentamos el caso de un edema agudo hemorrágico de aparición neonatal en una niña de 17 días de vida, valorada en nuestro servicio.

\section{Acute hemorrhagic edema in a newborn}

Key words:

- Acute hemorragic edema

- Vasculitis

- Henoch-Schönlein purpura

- Exanthema

- Neonate

Acute hemorrhagic edema of infancy is an infrequent disease with a benign course that appears between 3 months and 2 years old and, exceptionally, during the neonatal period. It is characterized by erythematous edema and purpuric lesions involving mainly the face and extremities, with a spontaneous recovery in days to weeks.

We report a case of acute hemorrhagic edema with a neonatal onset in a 17 days-old girl, followed in our service recently.
}

\section{INTRODUCCIÓN}

El edema agudo hemorrágico del lactante (EAHL) es una forma de vasculitis leucocitoclástica cutánea que aparece habitualmente en niños de edad comprendida entre los tres meses y los dos años, con ligero predominio en varones ${ }^{1}$.

La incidencia es desconocida, pero se asume que es infrecuente. Su aparición en el periodo neonatal, como el caso que nos ocupa, es excepcional.

\section{CASO CLÍNICO}

Presentamos el caso de una niña de diez días de vida, de origen chino, con fiebre de $38^{\circ} \mathrm{C}$ de tres horas de evolución, sin otra sintomatología. Alimentada con lactancia artificial. Sin ambiente epidémico familiar.

Como antecedentes personales, la gestación había mantenido controles normales; fue un parto eutócico sin factores de riesgo infeccioso, no precisó reanimación al nacimiento y las pruebas metabólicas estaban pendientes en ese momento.

Cómo citar este artículo: Checa Rodríguez R, Carabaño Aguado I, Álvarez Fernández B. Edema agudo hemorrágico en un neonato. Rev Pediatr Aten Primaria. 2015;17:151-3. 
A la exploración física presentaba buen estado general, sin aspecto séptico, siendo normal por aparatos.

En Urgencias se inicia protocolo de fiebre sin foco en paciente neonatal (analítica sanguínea, urinaria y de líquido cefalorraquídeo normales) y se pauta antibioterapia empírica.

Permanece afebril desde las 24 horas de ingreso y hasta el alta, sin incidencias salvo por aparición de exantema maculopapular al segundo día de ingreso, que remite de forma espontánea en las siguientes 24-48 horas. Ante la sospecha de viriasis, y una vez que se confirman los resultados definitivos de los cultivos (negativos), se suspende la antibioterapia. Se decide el alta a los 15 días de vida.

Dos días después, acude al Servicio de Urgencias por un exantema de un día de evolución, generalizado, a expensas de elementos "en diana" con aclaramiento central, sobreelevados y confluyentes. Leve coloración amoratada, más intensa en miembros inferiores, que se borra parcialmente a la presión, sin petequias (Figs. 1 y 2). Como única clínica acompañante, presentaba deposiciones líquidas escasas.

Se mantuvo en observación durante 24 horas y, ante la no progresión de las lesiones y el buen estado general, se decidió el alta.

Tras dos días en domicilio, acude nuevamente por shock hipovolémico secundario a un cuadro de diarrea abundante desde 18 horas antes, sin vómitos

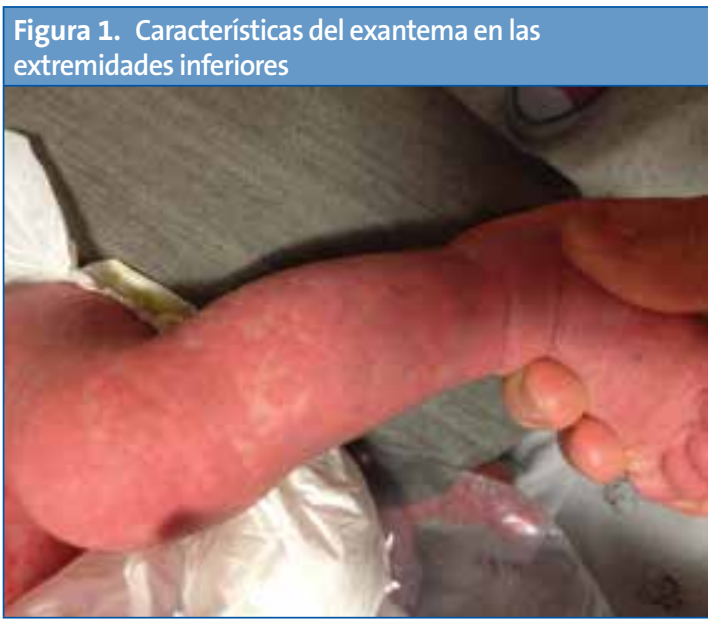

Figura 2. Características del exantema en el tronco

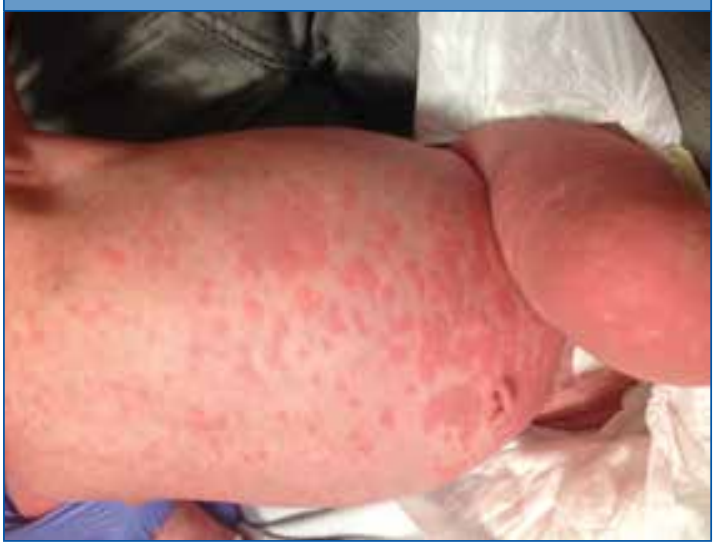

ni rechazo de tomas. Las lesiones cutáneas habían desaparecido. En el coprocultivo no se aisló germen y la virología fecal fue negativa. Recibió reposición con fluidoterapia intravenosa, que fue retirándose según mejoraba la tolerancia oral con leche hidrolizada y tras descartar afectación intestinal. Las deposiciones mejoraron progresivamente y se dio de alta asintomática.

En controles sucesivos en consulta ha presentado adecuada ganancia ponderoestatural, con deposiciones de consistencia normal. Mantuvo la alimentación con fórmula hidrolizada durante unas semanas, con posterior tolerancia de fórmula artificial, sin aparición de nuevas lesiones cutáneas.

\section{COMENTARIOS}

El edema agudo hemorrágico es una vasculitis leucocitoclástica que afecta especialmente a varones de entre tres meses y dos años de edad. Su presentación en el periodo neonatal, como el caso que nos ocupa, es infrecuente ${ }^{1}$. Se manifiesta con la aparición brusca de lesiones cutáneas extensas (1-5 cm), urticariformes, equimóticas, con tendencia a la expansión centrífuga, de distribución simétrica y de predominio en miembros inferiores y región facial, asociando edema. La etiología no se conoce completamente, pero la mayoría de casos documentados presentan un antecedente 
de infección, administración de fármacos o vacunas. En nuestro paciente, la relación causa-efecto se podría establecer con una infección gastrointestinal presumiblemente de etiología vírica.

Su evolución suele ser autolimitada, con resolución espontánea en 1-3 semanas, sin secuelas, pero puede presentar recurrencias, al igual que la púrpura de Schönlein-Henoch².

Los criterios diagnósticos se exponen en la Tabla $1^{3}$. Hasta en un tercio de los pacientes puede detectarse depósito de inmunoglobulina A (IgA) cuando se realiza biopsia cutánea ${ }^{4}$, aunque esta no es necesaria para el diagnóstico, que es clínico. Los ha-

Tabla 1. Criterios diagnósticos del edema agudo hemorrágico del lactante

Edad menor de dos años

Lesiones equimóticas y edema en cara, pabellones auriculares y miembros, con o sin afectación mucosa

Ausencia de afectación sistémica, con resolución espontánea en días-semanas

Fuente: Krause I, Lazarov A, Rachmel A. Acute hemorrhagicoedema of infancy, a benign variant of leucocytoclastic vasculitis. Acta Paediatr. 1996;85:114-7.

\section{BIBILIOGRAFÍA}

1. Savino F, Lupica MM, Tarasco V. Acute hemorrhagic edema of infancy: a troubling cutaneous presentation with a self-timing course. Pediatr Dermatol. 2013;30:149-52.

2. Ricart Campos S. Púrpura de Schönlein-Henoch. Protoc Diagn Ter Pediatr. 2014;1:131-40.

3. Krause I, Lazarov A, Rachmel A. Acute hemorrhagicoedema of infancy, a benign variant of leucocytoclastic vasculitis. Acta Paediatr. 1996;85:114-7. llazgos histopatológicos son idénticos a la púrpura de Schönlein-Henoch, lo que genera la controversia sobre si ambas entidades son diferentes o son manifestaciones extremas del mismo proceso ${ }^{5}$.

Debe realizarse el diagnóstico diferencial con otros procesos purpúricos como meningococemia, eritema multiforme o urticaria aguda con patrón hemorrágico.

Al tratarse de un cuadro benigno y autolimitado, se recomienda tratamiento sintomático, sin necesidad de otros tratamientos adicionales. Nuestra paciente evolucionó favorablemente, y fue dada de alta definitivamente, sin ninguna incidencia reseñable.

\section{CONFLICTOS DE INTERESES}

Los autores declaran no presentar conflictos de intereses en relación con la preparación y publicación de este artículo.

\section{ABREVIATURAS}

EAHL: edema agudo hemorrágico del lactante • IgA: inmunoglobulina $A$.
4. Puig Sanz L. Púrpuras. Protocolos de Dermatología. AEP, 2007. p. 201-7 [en línea] [consultado el 23/02/2015]. Disponible en www.aeped.es/sites/ default/files/documentos/purpuras.pdf

5. Cacharrón Caramés T, Díaz Soto R, Suárez García F, Rodríguez Valcárcel G. Edema hemorrágico agudo del lactante. An Pediatr (Barc). 2011;74:272-3. 